\title{
Neuroprotection: new pharmacological targets
}

\begin{abstract}
Recent research in the field of neuroprotection in glaucoma has focused on the $N$-methyl-D-aspartate (NMDA) and the $\alpha-2$ adrenergic receptor systems. Experimental findings have shown that brimonidine (BMD), a specific $\alpha-2$ adrenoceptor agonist, can protect against loss in mitochondrial membrane potential during oxidative stress, and preserve anterograde axonal transport. Memantine is a selective blocker of the NMDA-type glutamatergic type ion channel. It has unique open channel blocker properties that result in a preferential inhibition of excessive (excitotoxic) neuronal activation by high levels of glutamate without interfering with the channel's normal functions. In a primate model, memantine was neuroprotective as can be seen from its effect on maintaining/ preserving RGCs. Research in Alzheimer's disease has demonstrated that memantine may increase neuronal functionality as well as being protective. Research in a primate glaucoma model shows that memantine protects neurons from shrinking in the lateral geniculate nucleus. Memantine is currently in clinical trials for glaucoma.
\end{abstract}

Eye (2007) 21, S46-S50; doi:10.1038/sj.eye.6702889

Keywords: $\alpha-2$ receptors; NMDA receptors; memantine; brimonidine; neuroprotection; glaucoma

Biological Sciences, Research and Development, Allergan Inc., Irvine, USA

Correspondence: L Wheeler, Biological Sciences, Research and Development, Allergan Inc., Irvine, USA Tel: + 17142464447 . E-mail: Wheeler_Larry@ Allergan.com

\section{Introduction}

Research in the field of neuroprotection in glaucoma has been focused on drugs that can either enhance neuronal survival or block compounds that may cause cell death. Selective $\alpha-2$ adrenergic agonists and the $\mathrm{N}$-methyl-D-aspartate (NMDA) receptor blockers are examples of pharmacological agents of the aforementioned strategies to protect retinal ganglion cells (RGCs) in glaucoma that are in clinical testing. The highlights of key findings and recent discoveries, with an emphasis on the NMDA receptor system, are provided here.

The $\alpha-2$ adrenergic receptor system

Adrenergic receptors mediate the biological effects of the sympathetic nervous system. To date, nine distinct $\alpha$ - and $\beta$-adrenergic receptor subtypes have been cloned from several species. $\alpha$-Adrenergic receptors are subdivided into the $\alpha-1$ and $\alpha-2$ subgroups. ${ }^{1,2}$ The precise physiological functions and therapeutic potential of many of these receptors have not been fully elucidated. However, selective agonists for the $\beta$-2 adrenergic receptor have been developed for use in asthma therapy, and $\alpha-1$ receptor antagonists are first-line medication for patients with hypertension, coronary heart disease, or chronic heart failure. So far, three distinct genes have been identified from several species that encode for the three subtypes of $\alpha-2$ adrenergic receptors, $\alpha-2 a,-2 b$, and $-2 c{ }^{1}{ }^{1}$ These genes have highly homologous amino-acid sequences, thus making the generation of subtype selective antibodies difficult. Mapping the location of the $\alpha-2$ subtypes in the eye has been problematic. However, a new set of antibodies has been successfully made. These selective antibodies have allowed the location of the $\alpha-2$ adrenergic receptors in the retina to be identified. The $\alpha-2 \mathrm{a}$ antibody preferentially binds to RGCs, while $\alpha-2 b$ is much more distributed in the retina, resembling Müller cell staining to some extent. $\alpha$-2c is almost exclusively located in the photoreceptor layer. ${ }^{3}$

There are multiple injuries to the RGCs and surrounding cells in glaucoma, ${ }_{1}^{4}$ offering a wide scope of opportunities for neuroprotective therapies. The mechanisms of action of $\alpha-2$ agonists and their signal transduction have generated interest, because their understanding may lead to new pharmacological targets. 
Involvement of $\alpha-2$ adrenergic receptors in the neuroprotective signalling pathway

The mechanisms underlying the neuroprotective signalling pathway involving $\alpha-2$ adrenergic receptors are not fully elucidated. One issue is that RGCs have to be highly differentiated to express $\alpha-2$ receptors. Thus, neuroprotective pathways of $\alpha-2$ adrenergic receptors cannot be easily studied in RGCs. This problem was overcome by studying transfected cell lines that overexpress $\alpha$-2 receptors. Using these cell lines, we were able to demonstrate biochemically and functionally that the activation of mitogen-activated protein kinase (MAPK) and Akt kinase (protein kinase B) is involved in neuroprotective signalling by selective $\alpha-2$ agonists such as brimonidine (BMD) (L Wheeler et al, private communication).

Specific $\alpha-2$ adrenergic receptor protection against oxidative stress has also been demonstrated at the level of the mitochondrion, which is a cellular component having a key role in RGC apoptosis. We found that $\alpha-2$ agonists can send signals that help to protect the mitochondria during oxidative stress. In this study, cells overexpressing $\alpha-2$ adrenoceptors were loaded with JC-1 dye, a dye that is used for monitoring the mitochondrial transmembrane potential (see R Funk's presentation). When oxidative stress was induced by exposing cells to ultraviolet light in the presence or absence of BMD, loss in mitochondrial transmembrane potential induced by the oxidative stress was prevented by BMD (Figure 1).
Other studies support the hypothesis that there are signalling systems in the retina that help cells under stress. Survival factors, such as brain-derived neurotrophic factor, signal through these pathways (Figure 2). Research findings suggest that $\alpha-2$ agonists may be an additional, or stress induced, system that helps cells deal with localized stress (Figure 2). 5,6

\section{The NMDA receptor system}

Numerous studies have contributed to our understanding of NMDA receptor function. ${ }^{7}$ Under physiological conditions, the NMDA channel is blocked by magnesium ions unless activity in other classes of excitatory synaptic inputs depolarizes the neuronal membrane sufficiently to relieve this block. ${ }^{8}$ When neurons are injured, the neuronal membrane may become chronically depolarized. Under these conditions, even normal levels of extracellular glutamate will result in excessive activation of NMDA channels and a consequent overload of intracellular $\mathrm{Na}^{+}$and $\mathrm{Ca}^{2+}$ ions, which flow from the extracellular space through the open NMDA channels and into the neuronal cytoplasm. Elevated cytoplasmic levels of $\mathrm{Na}^{+}$and $\mathrm{Ca}^{2+}$ ions leads to further neuronal damage and mediates the neuronal injury associated with glutamatergic excitotoxicity. ${ }^{7}$

Memantine behaves as an open-channel blocker at the NMDA channel, that is glutamate must first bind to its receptor site and open the channel before memantine can access its binding site within the channel. Thus, for a

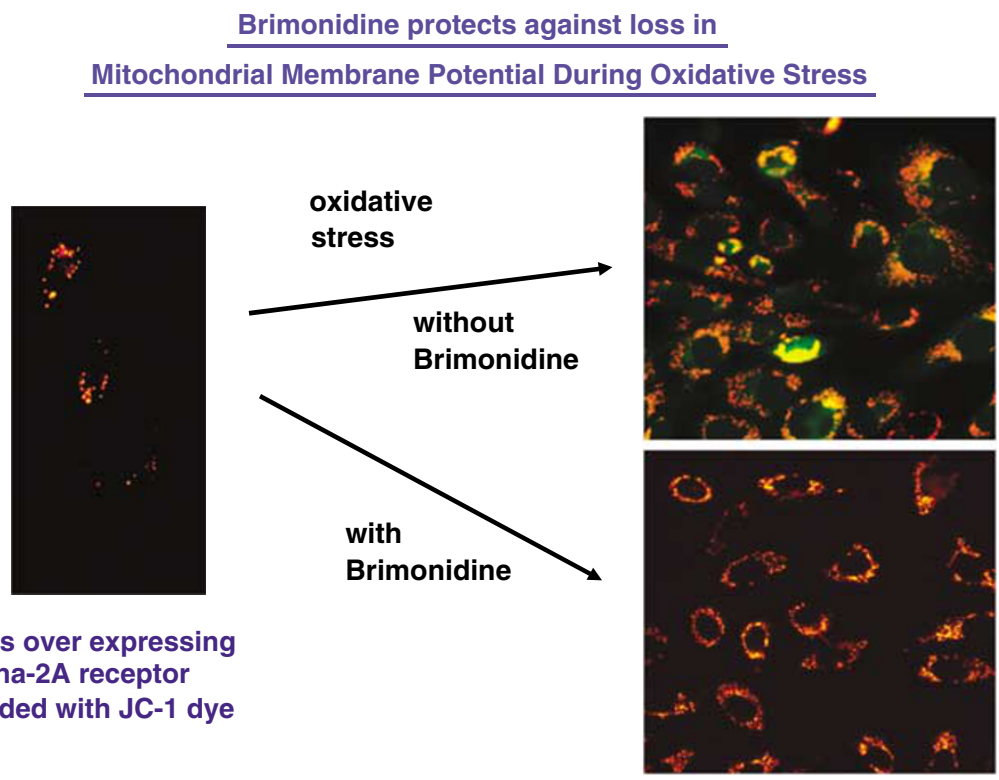

Figure 1 Protection by $\alpha-2$ adrenergic receptor agonist against loss in mitochondrial membrane potential during oxidative stress. BMD was present at $500 \mathrm{nM}$ in cells overexpressing $\alpha-2$ adrenergic receptors; yellow/green colours show cells in which the mitochondrial membrane potential has dropped and apoptosis is about to proceed. 


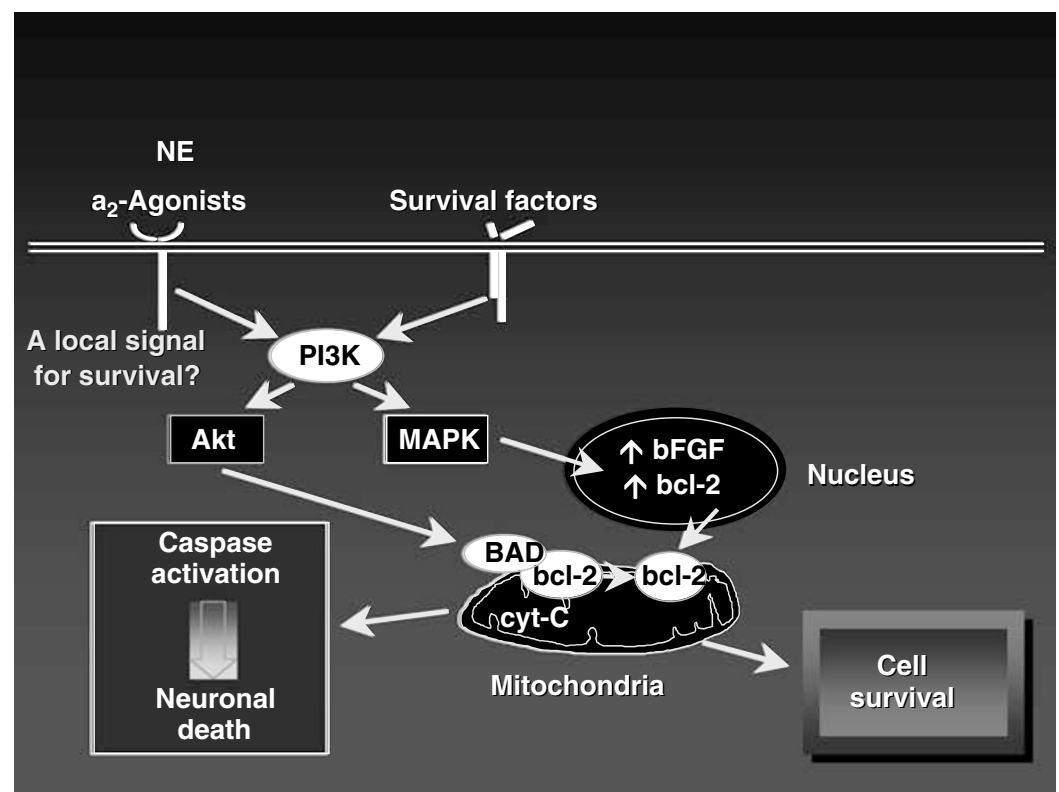

Figure 2 Survival pathways activated by $\alpha-2$ adrenoceptor agonists. Ongoing research has suggested that activation of $\alpha$-2-adrenergic receptors by BMD leads to activation of the same intracellular signaling pathway that neurotrophic factors use to promote neuronal survival. Both BMD and neurotrophic receptors cause activation of phosphatidylinositol-3-kinase (PI3 K), leading to activation of the MAPK cascade and Akt. The MAPK and Akt pathways have many survival-promoting effects, including the induction of the antiapoptotic protein bcl-2. Activated Akt phosphorylates the proapoptotic protein BAD, leading to its dissociation from bcl-2 and the inhibition of apoptotic activity, which involves cytochrome $c$ release from the mitochondria. Cytochrome $c$ interacts with cytosolic proteins, resulting in the activation of caspases, the proteases responsible for the execution of the apoptotic program and apoptotic neuronal death. (Reproduced from Weinreb RN (ed.) Glaucoma Neuroprotection. Wolters Kluwer Health, Philadelphia. pp 56. Copyright 2006, with permission from Lippincott Williams \& Wilkins.)

given fixed concentration of memantine, the fractional block of NMDA channels will increase with increasing extracellular glutamate concentration. This results from the fact that, as the concentration of glutamate is increased, the channels will spend proportionately more time in the open state and the level of memantine block will increase. This property of open-channel blockade, in combination with other properties of binding affinity and binding kinetics, makes memantine relatively more effective to block the excessive levels of NMDA channel activity associated with pathological conditions, while having relatively less effect to block the lower levels of channel activation associated with normal physiological neurotransmission. The favourable tolerability and low drop-out rate observed in Alzheimer's disease trials using memantine treatment are consistent with these

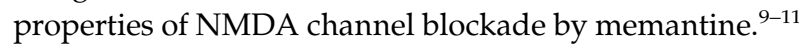

In an ocular hypertensive rat model, memantine, a compound which does not lower intraocular pressure (IOP), has been found to prevent the progression of RGC loss subsequent to laser-induced IOP elevation. ${ }^{12}$ In a study of chronic ocular hypertension in the primate, histological measurements showed that, for animals with moderate elevation of IOP, oral administration of memantine was associated with an enhanced survival of
RGCs in the inferior retina. ${ }^{13,14}$ Measurements of optic nerve head topography with confocal scanning laser tomography (HRT) showed less IOP-induced change in animals treated with memantine, as reflected in measurements of the cup and neuroretinal rim (Figure 3). In addition, the findings indicate that memantine treatment is safe. One of the more interesting results was obtained from recordings of the visual evoked potential (VEP). When responses from animals with severe RGC loss were compared, vehicle-treated animals had lost most of the VEP response while responses obtained from memantinetreated animals were of relatively normal amplitude. ${ }^{13}$

Further research has focused on whether memantine increases neuronal functionality, in some way, in addition to its neuroprotective effects. A neurological study showed that memantine increases brain metabolism and effectively treats mild-to-moderate Alzheimer's disease. ${ }^{9,10}$ In a phase III study, patients with Alzheimer's disease were treated with memantine, up to $20 \mathrm{mg}$ a day, for 24 weeks. ${ }^{18} \mathrm{~F}$-deoxyglucose labelling was measured in patients while they identified pictures on a monitor. A positron emission tomography (PET) scanner was used to construct a picture of metabolic activity. In the regions commonly associated with Alzheimer's disease, there was a decrease in glucose uptake in the control group, 

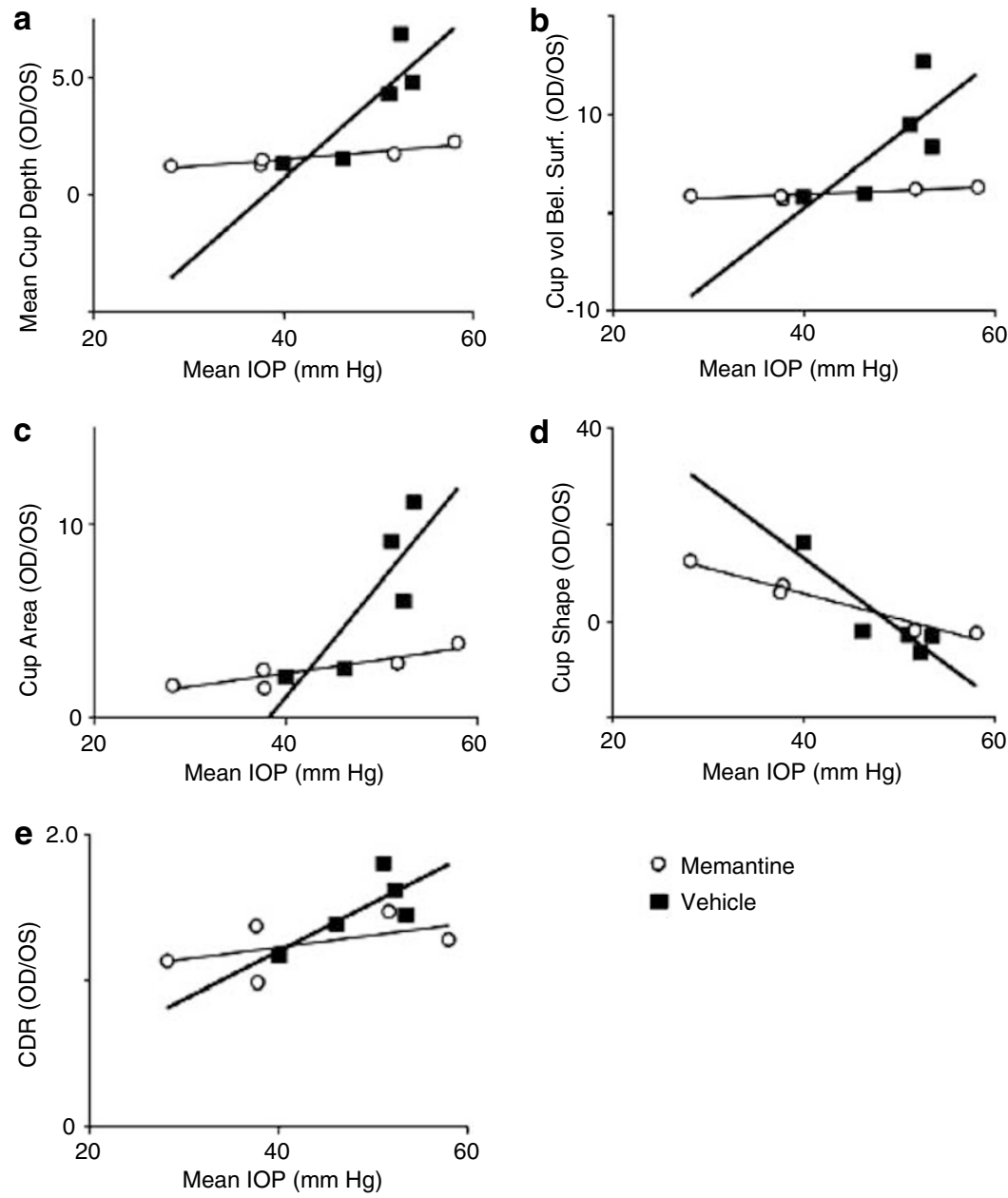

- Memantine

- Vehicle

Figure 3 Summary of normalized cup measurements from confocal laser scans of the five animals having the highest mean IOP in each treatment group. Values are plotted as a function of mean IOP for measurements of (a) mean cup depth, (b) cup volume below surface, (c) cup area, (d) cup shape and (e) global cup/disc ratio. In each panel, linear regression is plotted as a heavy line for vehicletreated animals (filled squares) or a light line for memantine-treated animals (open circles). (Reproduced from Hare $\mathrm{et}^{\mathrm{al}} \mathrm{l}^{14}$ with permission of Invest Ophthalmol Vis Sci via Copyright Clearance Center.)

whereas a very robust uptake was noted in the memantine-treated patients. ${ }^{15}$

Yucel et $a l^{16}$ have recently been able to show that memantine protects neurons from shrinking in the lateral geniculate nucleus (LGN) in experimental glaucoma in the monkey. LGN relay neurons (layers 1, 4, and 6) were examined following parvalbumin immunolabelling. Analysis of covariance results showed significantly less neuron shrinkage in the memantine-treated group for layers 1,4 , and $6(P<0.001, P<0.02$, and $P<0.04$, respectively). This difference was highest in layer 1 . In each of these layers, neuron numbers did not differ significantly between groups. These results show that memantine treatment was associated with prevention of neuronal atrophy in higher levels of the glaucoma-injured visual pathway and are consistent with the earlier report of functional preservation based on
VEP recordings from memantine-treated monkeys with experimental glaucoma.

\section{Conclusion}

Memantine can protect at multiple levels of the visual pathway. The finding that memantine protects adult visual neurons from transsynaptic atrophy in experimental glaucoma could be of therapeutic value. Currently, memantine is being tested in an ongoing clinical trial as a treatment for glaucoma.

\section{References}

1 Bylund DB, Eikenberg DC, Hieble JP, Langer SZ, Lefkowitz RJ, Minneman KP et al. International Union of 
Pharmacology nomenclature of adrenoceptors. Pharmacol Rev 1994; 46: 121-136.

2 Kintsurashvili E, Gavras I, Johns C, Gavras H. Effects of antisense supraspinal alpha 1 adrenoceptors mediate pronociception. Anesthesiology 2002; 96: 367-374.

3 Woldemussie E, Wijono M, Ruiz G. Muller cell response to laser-induced increase in intraocular pressure in rats. Glia 2004; 47: 109-119.

4 Levin LA. Retinal ganglion cells and neuroprotection for glaucoma. Surv Ophthalmol 2003; 48(Suppl 1): s21-s24.

5 Lai R, Chun T, Hasson D, Lee S, Mehrbod F, Wheeler L. Alpha-2 adrenoceptor agonist protects retinal function after acute retinal ischemic injury in the rat. Vis Neurosci 2002; 19: 175-185.

6 Wheeler L, WoldeMussie E, Lai R. Role of alpha-2 agonists in neuroprotection. Surv Ophthalmol 2003; 48(Suppl 1): S47-S51.

7 Lipton SA. Failures and successes of NMDA receptor antagonists: molecular basis for the use of open-channel blockers like memantine in the treatment of acute and chronic neurologic insults. NeuroRx 2004; 1(1): 101-110.

8 Nowak L, Bregestovski P, Ascher P, Hebet A, Prochiantz A. Magnesium gates glutamate-activated channels in mouse central neurones. Nature 1984; 307: 462-465.

9 Bullock R. Efficacy and safety of memantine in moderate-to-severe Alzheimer disease: the evidence to date. Alzheimer Dis Assoc Disord 2006; 20(1): 23-29.
10 Orgogozo JM, Rigaud AS, Stoffler A, Mobius HJ, Forette F. Efficacy and safety of memantine in patients with mild to moderate vascular dementia: a randomized, placebocontrolled trial (MMM 300). Stroke 2002; 33(7): 1834-1839.

11 Chen HS, Lipton SA. The chemical biology of clinically tolerated NMDA receptor antagonists. J Neurochem 2006; 97(6): 1611-1626.

12 WoldeMussie E, Yoles E, Schwartz M, Ruiz G, Wheeler LA. Neuroprotective effect of memantine in different retinal injury models in rats. J Glaucoma 2002; 11(6): 474-480.

13 Hare WA, Woldemussie E, Ruiz L, Wheeler L. Efficacy and safety of memantine treatment for reduction of changes associated with experimental glaucoma in the monkey, I: functional measures. Invest Ophthalmol Vis Sci 2004; 45 2625-2639.

14 Hare WA, WoldeMussie E, Weinreb RN, Ton H, Ruiz G, Wijono $\mathrm{M}$ et al. Efficacy and safety of memantine treatment for reduction of changes associated with experimental glaucoma in monkey, II: structural measures. Invest Ophthalmol Vis Sci 2004; 45(8): 2640-2651.

15 Potkin S, McDonald S, Gergel I, Alva G, Keater D, Fallon J. Memantine monotherapy increases brain metabolism (PET) and effectively treats mild to moderate Alzheimer's disease. Eur J Neurol 2004; 11(Suppl 2): 44.

16 Yucel YH, Zhang Q, Gupta N, Kaufman PL, Weinreb RN. Loss of neurons in magnocellular and parvocellular layers of the lateral geniculate nucleus in glaucoma. Arch Ophthalmol 2000; 118(3): 378-384. 